\title{
Planificación turística sostenible: las comunidades de Santa Elena.
}

Sustainable tourism planning: the communities of Santa Elena.

Yumisaca Tuquinga Jhony Ernesto. ${ }^{1}$, Delgado Castro Alejandro. ${ }^{2}$, Pérez Chiquito Maritza Marianela. ${ }^{3}$ \& Mendoza Tarabó Arnaldo Efrén. ${ }^{4}$

\section{Resumen.}

La planificación turística en comunidades del litoral Santa Elenense en Ecuador requiere de un enfoque que responda a la realidad de espacios rurales ancestrales de uso común, que considere la diversidad de actores y los patrimonios culturales y naturales, como elementos significantes de sostenibilidad. En este contexto, la presente investigación identifica los principales lineamientos usados en la planificación turística de Santa Elena, prestando atención a la necesidad de alcanzar el desarrollo local, la competitividad turística y la sostenibilidad de sus patrimonios mediante otros enfoques. El trabajo incluyó desde una investigación documental a trabajos con actores del turismo y académicos, mediante la técnicas de la encuesta y el focus group, permitiendo como resultados identificar ejes estratégicos que se enfocan bajo una perspectiva top-down y una necesidad de alcanzar el desarrollo sostenible mediante aspectos fundamentales con un enfoque inverso (bottom-up).

Palabras claves: Planificación turística, desarrollo local, sostenibilidad, Santa Elena.
Abstract.
Tourism planning in Santa Elena coastal communities in Ecuador requires an approach that responds to the reality of ancestral rural spaces of common use, which considers the diversity of actors and cultural and natural heritage, as significant elements of sustainability. In this context, this research identifies the main guidelines used in Santa

\footnotetext{
${ }^{1}$ Universidad Estatal Península de Santa Elena. Santa Elena, Ecuador.jyumisaca@ upse.edu.ec

${ }^{2}$ Universidad de la Habana - Cuba. alejandro_delgado@ftur.uh.cu

${ }^{3}$ Universidad Estatal Península de Santa Elena. Santa Elena, Ecuador.mperez@upse.edu.ec

${ }^{4}$ Universidad Estatal Península de Santa Elena. Santa Elena, Ecuador. emendoza@ upse.edu.ec
} 
Elena's tourism planning, paying attention to the need to achieve local development, tourism competitiveness and the sustainability of its heritage through other approaches. The work included from a documentary investigation to works with actors of the tourism and academic, by means of the techniques of the survey and the focus group, allowing like results to identify strategic axes that are focused under a top-down perspective and a need to reach the sustainable development through fundamental aspects with a reverse (bottom-up) approach.

Keywords: Tourism planning, local development, sustainability, Santa Elena.

\section{Introducción.}

El turismo indiscutiblemente puede contribuir al desarrollo de los pueblos, por su constante crecimiento, dinamismo económico y fundamentalmente por la relación entre el visitante y la población local que surge de la actividad; de hecho, Denman (2006) menciona que la actividad turística conlleva a tres aspectos importantes: interacción entre los visitantes, las comunidades anfitrionas y sus entornos locales, sensibilización de los visitantes y anfitriones de los problemas ambientales y dependencia del turismo a la exigencia de los visitantes por entornos intactos y limpios, zonas naturales atractivas, tradiciones históricas y culturales auténticas y gentes acogedoras con las que puedan tener una buena relación [1].

El interés de la demanda por lo natural, por la aventura y por una convivencia cultural, genera oportunidades de diversificación y dinamismo económico para las comunidades rurales en general y para el sector turístico en particular. En este contexto, es factible para las comunidades mejorar su competitividad ofreciendo servicios de calidad y ofertas complementarias; aunque el turismo implica planificación e innovación constante, el primer aspecto normalmente es la base de la funcionalidad y éxito del destino y a él se dedica precisamente este trabajo.

En ocasiones, en el ámbito turístico surge la controversia sobre el daño que el turismo puede provocar, sin embargo, Plumed (2018) manifiesta que esto ocurre cuando existe una falta de planificación [2]; por tanto, Popovich (2006) asegura que una buena planificación requiere considerar a la población del territorio, y gestionar los impactos del turismo, de modo que éste promueva el desarrollo socioeconómico del área y su población [3]. Para lograr una actividad sostenible en los destinos turísticos, Vera (2001) aduce que se debe inducir la praxis y operatividad del marco institucional y legal, así como los principios de planificación [4].

La complejidad de la planificación en la práctica se debe a la dificultad en el entendimiento del concepto desde el enfoque de sostenibilidad, a la imposición de actividades por actores sociales y a la priorización económica sobre los demás ejes, de hecho, según Garcìa (2014) el desarrollo turístico y la sostenibilidad pueden coexistir siempre que haya una adecuada estrategia de protección sin descuidar las aspiraciones de la población local y de los visitantes [5]. Lo expuesto indica que la planificación es un 
proceso complejo, donde se conjugan diversos factores, tal como asegura Garcìa (2003), la planificación establece medidas apropiadas para que el rendimiento revierta en el territorio y en el sector turístico, constituyéndose como un elemento dinamizador y generador de rentas complementarias a las frágiles economías agropecuarias típicas del medio rural [6].

La planificación turística no responde a un método único y menos a un diseño común de aplicación, ésta varía inevitablemente según las características del territorio, los métodos y sobre todo los objetivos; Inskeep (1991) asegura que es un proceso continuo con suficiente flexibilidad para permitir la adaptación a las circunstancias cambiantes de los destinos [7]. La planificación en esencia, según López (1997) es un ejercicio de participación y consenso de todas las fuerzas económicas y sociales con capacidad de decisión e inversión en la mejora del ciclo de vida del producto y la competitividad del territorio turístico [8].

Harvey (2012) menciona que en la contemporaneidad se acusa a la planificación tradicional de usar métodos sofisticados de decisión top-down, influenciada por fuertes intereses sectoriales y económicos, imponiendo en forma tecnocrática y política cambios importantes en el espacio y ecosistemas de vida de las poblaciones [9]. Ante esta realidad, Baque (2011) citado por Goñi (2014) menciona que el nacimiento y consolidación de planificaciones construidas colectiva y colaborativamente de abajo hacia arriba, toma fuerza en momentos en que las formas tradicionales de gobierno y organización colectiva de la sociedad, así como de la planificación, entran en crisis [10].

Este último enfoque cobra importancia por la poca atención a los aspectos culturales y de identidad, así como el escaso protagonismo de los habitantes en el proceso de planificación; Beebeejaun (2012) asevera que en la actualidad el trabajo colectivo está dando como resultado una renovación de la planificación y de su capacidad de pensar los territorios rurales como un conjunto de historias, valores y vínculos entre los habitantes y su ambiente [11]. La planificación turística demuestra una fuerte tensión entre el rol que los organismos de planificación desean tener y la fuerte resistencia de las tradiciones locales, siendo el momento de considerar la voluntad de las comunidades.

Planificar el turismo en la provincia de Santa Elena en Ecuador resulta primordial, ya que, según el Gobierno Provincial Autónomo Descentralizado de Santa Elena (2015) el turismo es una actividad muy importante para la localidad, debido al potencial que el territorio posee, además de ser una actividad de la que se derivan otras relacionadas al comercio, artesanías y oferta de servicios varios [12]. Para el Gobierno Autónomo Descentralizado del Cantón Santa Elena (2014), las actividades de mayor desarrollo se dan en el área rural, sobretodo en el sector primario y en menor proporción en el sector servicios [13]; pese a esta aseveración, el turismo carece de una planificación propia para su desarrollo. 
Las actividades que se desarrollan en este campo responden a la implicación de emprendedores en respuesta a la llegada de los visitantes, más no a una planificación que contribuya al desarrollo de las localidades; Diéguez (2015) manifiesta que existen notables posibilidades de planificar el turismo y alcanzar su sostenibilidad siempre y cuando se enfoque a los interés de la población [14]. En este sentido, el objetivo de este artículo es identificar las líneas estratégicas que deben abordar las planificaciones turísticas en el contexto actual santaelenense, concretando en aquellos aspectos de interés externos e internos y la manera de abordarlos desde las perspectivas de la sostenibilidad.

\section{Metodología.}

Para lograr el objetivo propuesto se implementó un proceso metodológico estructurado en tres fases. En primera instancia se enfatizó en la investigación documental, identificando instrumentos de planificación formulados con el fin de conocer sus objetivos y la relación con la sostenibilidad, entre ellos: el plan de desarrollo provincial y los planes de desarrollo de los municipios de Santa Elena, La Libertad y Salinas; documentos de trabajos de titulación de las carreras de Gestión y Desarrollo Turístico y de Hotelería y Turismo de la Universidad Península de Santa Elena. Esta información relevante fue vaciada en fichas síntesis, donde resaltan sus dimensiones y los enfoques estratégicos.

En un segundo momento se trabajó en analizar la situación del turismo de Santa Elena, para lo cual se usó la técnica de focus-group con académicos del ramo, cuyos objetivos fueron generar discusión sobre la situación del turismo en la provincia y determinar la concordancia de estos expertos sobre la necesidad de generar una planificación turística sostenible para las comunidades rurales en el litoral santaelenense. Los resultados fueron plasmados en un FODA para el primer caso y en una matriz de concordancia para el segundo caso.

Finalmente, en la tercera fase se recogieron opiniones de actores del turismo de la provincia mediante entrevistas en profundidad, entre ellos, representantes de entidades gubernamentales, representante de la cámara de turismo y de la federación de comunas, dirigentes de comunas que trabajan en turismo y un representante de las juntas parroquiales. Las preguntas planteadas pretendieron la aceptación o el rechazo a la necesidad de planificar el turismo en las comunidades y que estas planificaciones respondan a las necesidades locales, por tanto, tenga su origen en las bases y aliente a la participación de su desarrollo. Los resultados plasmados orientan a abordad la planificación turística desde líneas estratégicas resultantes del análisis de 29 aspectos clave y una plataforma necesaria de sostenibilidad y competitividad.

\section{Análisis y Resultados.}

Planificación del turismo en la Provincia de Santa Elena. 
En la provincia de Santa Elena en los últimos seis años se han formulado ocho planes de desarrollo turístico a nivel comunal, un plan de desarrollo provincial que acoge ciertos lineamientos de enfoques económicos en la cual se incluye al turismo, tres planes de desarrollo cantonal que consideran el eje turístico dentro de sus programas, nueve planes de desarrollo parroquial con enfoques de producción de acorde a su potencialidad existente y un plan de desarrollo endógeno comunal que aborda al turismo como eje estratégico principal de desarrollo local.

Los planes formulados a nivel comunal corresponden a trabajos de titulación y uno corresponde al resultado de una consultoría de la empresa pública, estos evidencian un enfoque participativo, lo que marca un cierto grado de diferenciación con el resto de propuestas, que desde luego tienen cierto direccionamiento a intereses sectoriales y económicos. El plan de desarrollo provincial formulado mediante licitación pública evidencia enfoques mínimos hacia el sector turísticos y resulta muy pobre en conceptos de sostenibilidad; en lo que respecta a los planes de desarrollo cantonal, en sus tres casos (Salinas, La Libertad y Santa Elena) fueron ejecutados mediante consultoría privada, aunque estas tienen enfoques de turismo entre los eje de desarrollo local, existen programas muy incipientes que no consideran la sostenibilidad, inclusión, ni aspectos de competitividad. Finalmente, en lo que respecta a los nueve planes parroquiales, estos consideran programas concretos de turismo para aquellas comunidades en etapas de implicación y desarrollo dentro del ciclo de vida, desconsiderando acciones para aquellas comunidades que aún no han incursionado en la actividad pese a sus potencialidades.

Según Cornejo (2009) el análisis de los planes determina si estos consideran criterios relacionados con los conceptos, dimensiones y objetivos de una actividad sustentable [15], por tanto, a partir de este marco conceptual se han determinado los criterios para analizar la sustentabilidad de los planes en Santa Elena. La tabla siguiente muestra los resultados sobre criterios de sustentabilidad en los planes de desarrollo analizados, señalando con una $\mathrm{T}$ la incorporación total del criterio y con una $\mathrm{P}$ la incorporación parcial; además se puede apreciar que hay criterios que definitivamente no se utilizan en ninguno de los planes estudiados.

Tabla 1 Criterios de sostenibilidad y aspectos de interés en los planes de desarrollo en Santa Elena.

\begin{tabular}{|l|l|c|c|c|c|}
\hline NNo & \multicolumn{1}{|c|}{ CRITERIOS } & $\begin{array}{c}\text { Plan de } \\
\text { desarrollo } \\
\text { provincial } \\
2015-2019\end{array}$ & $\begin{array}{c}\text { Plan de } \\
\text { desarrollo } \\
\text { cantonal } \\
2014-2019\end{array}$ & $\begin{array}{c}\text { Plan de } \\
\text { desarrollo } \\
\text { parroquial } \\
2014-2019\end{array}$ & $\begin{array}{c}\text { Plan de desarrollo } \\
\text { endógeno comuna } \\
\text { Dos Mangas } \\
2015-2017\end{array}$ \\
\hline 1 & $\begin{array}{l}\text { La sustentabilidad debe aplicarse a todo tipo } \\
\text { de turismo }\end{array}$ & $\mathrm{P}$ & $\mathrm{T}$ \\
\hline 2 & $\begin{array}{l}\text { Equilibrio adecuado: medioambiental, } \\
\text { económico y sociocultural }\end{array}$ & $\mathrm{P}$ & $\mathrm{P}$ & $\mathrm{P}$ & $\mathrm{P}$ \\
\hline 3 & $\begin{array}{l}\text { Medio ambiente natural, cultura local y } \\
\text { residentes locales. }\end{array}$ & $\mathrm{P}$ & $\mathrm{P}$ & $\mathrm{P}$ \\
\hline 4 & $\begin{array}{l}\text { Planificación pertinente con planes nacional, } \\
\text { regional y local }\end{array}$ & $\mathrm{P}$ & $\mathrm{P}$ & $\mathrm{P}$ & $\mathrm{P}$ \\
\hline 5 & $\begin{array}{l}\text { Planificación: resultado de proceso } \\
\text { participativo e informado de todos los actores }\end{array}$ & $\mathrm{P}$ & $\mathrm{P}$ & $\mathrm{P}$ & $\mathrm{T}$ \\
\hline 6 & Enfoque integrador & $\mathrm{P}$ & $\mathrm{P}$ & $\mathrm{P}$ & $\mathrm{P}$ \\
\hline
\end{tabular}


ISSN 2661-6831

Vol. 3 No. 3 pág. 38-50, Jul-Sep. 2019

www.exploradordigital.org

\begin{tabular}{|c|l|c|c|c|c|}
\hline \hline 7 & $\begin{array}{l}\text { Conocimiento de exigencias de demanda } \\
\text { Experiencia significativa en turistas para } \\
\text { hacerlos conscientes en problemas de } \\
\text { Sostenibilidad }\end{array}$ & & $\mathrm{P}$ & & $\mathrm{P}$ \\
\hline 9 & $\begin{array}{l}\text { Actitud vigilante, mecanismo de supervisión y } \\
\text { sistema de indicadores de técnicas de }\end{array}$ & $\mathrm{P}$ & $\mathrm{P}$ & $\mathrm{P}$ & $\mathrm{P}$ \\
\hline 10 & $\begin{array}{l}\text { Mayor adaptación de } \\
\text { planificación, gestión y seguimiento }\end{array}$ & & & \\
\hline 11 & Viabilidad Económica & & & $\mathrm{P}$ & $\mathrm{T}$ \\
\hline 12 & Prosperidad Local & $\mathrm{P}$ & $\mathrm{P}$ & $\mathrm{P}$ & $\mathrm{P}$ \\
\hline 13 & Calidad de Empleo & & & $\mathrm{P}$ & $\mathrm{P}$ \\
\hline 14 & Justicia Social & & & $\mathrm{P}$ & $\mathrm{P}$ \\
\hline 15 & Control Local & & & & $\mathrm{P}$ \\
\hline 16 & Satisfacción de todo tipo de visitante & & & $\mathrm{P}$ & $\mathrm{P}$ \\
\hline 17 & Bienestar de la comunidad & & $\mathrm{P}$ & $\mathrm{P}$ & $\mathrm{P}$ \\
\hline 18 & Riqueza cultural & $\mathrm{P}$ & $\mathrm{P}$ & & $\mathrm{P}$ \\
\hline 19 & Integridad Física & & & $\mathrm{P}$ & $\mathrm{P}$ \\
\hline 20 & Diversidad Biológica. & & & & $\mathrm{P}$ \\
\hline 21 & Eficiencia de Recursos & $\mathrm{P}$ & $\mathrm{P}$ & $\mathrm{P}$ & \\
\hline 22 & Pureza Ambiental & &
\end{tabular}

Fuente: Elaboración propia a partir de criterios de sostenibilidad propuestos por Cornejo 2009.

A excepción de los criterios uno, cinco y once, que incorporan en su totalidad (T) las variables que estipulan dichos criterios y que se identifican en el plan de desarrollo endógeno de Dos Mangas, en su mayoría los planes alcanzan parcialmente (P) los criterios, evidenciando además, que el uso de tecnologías (criterio 10) no está presente en ninguno de los planes; al igual que los criterios $14,15,16$ y 17 , no son considerados en la planificación provincial y cantonal, lo que demuestra que los planes de desarrollo presentados para la provincia de Santa Elena tienen objetivos encaminados al cumplimiento de directrices de organismos públicos, lo que implica que estamos frente a planificaciones con enfoque top-down.

\section{Situación del turismo en la provincia de Santa Elena: necesidad de generar una planificación sostenible.}

Según el Ministerio de Turismo de Ecuador - MINTUR (2017), Santa Elena reconocida por su gran extensión de playa y la dedicación a la actividad turística encausada en el modelo tradicional de sol y playa, recibió 425.406 visitantes al finalizar el 2016, lo que implica un 30\% de los traslados nacionales [16]. No obstante, son los principales feriados en al año los que inducen la movilidad hacia este destino, lo que implica que esta provincia tiene una marcada estacionalidad, aún así, la actividad turística es uno de los principales ejes de desarrollo económico, aunque este llamado desarrollo está lejos de beneficiar equitativamente a su población y de generar una mayor conciencia ambiental y cultural. La siguiente tabla sintetiza en un anàlisis FODA la realidad del turismo en esta provincia.

Tabla 2 Análisis FODA del turismo en la provincia de Santa Elena. 


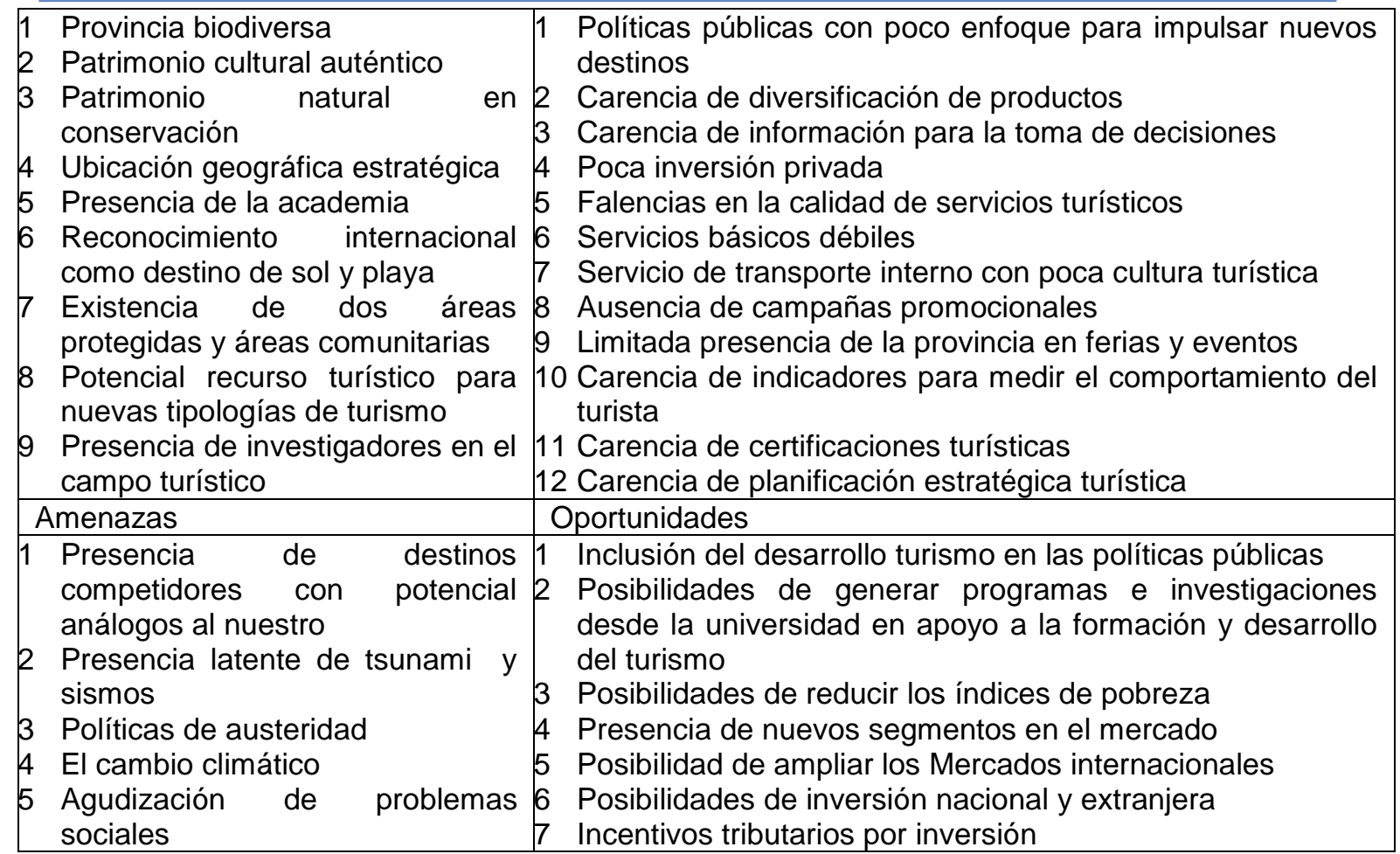

Fuente: Elaboración propia a partir del trabajos de focus group. 2018

Siguiendo a Ponce (2006) que demuestra que de la Matriz FODA se desprenden nuevas matrices que permite el desarrollo del marco analítico y las estrategias [17], se realizó una evaluación de los factores internos y externos (MEFI - MEFE), obteniendo como resultados que las fuerzas internas son favorables para el destino, con un peso ponderado de 1.51 contra 1.28 de las debilidades; mientras tanto, el peso ponderado de las oportunidades fue de $2.19 \mathrm{y}$ de las amenazas de 0.82 , lo cual establece que el medio ambiente externo es también favorable para el destino Santa Elena.

Finalmente se desarrolló el análisis estratégico del FODA, resaltando en este punto las principales estrategias resultantes del cruce de variables FO, DO, FA y DA, pudiendo realizarse interesantes observaciones. En el caso de (FO) se denota que es el más fuerte ya que se integra las fortalezas y las oportunidades con que cuenta el destino, y el cuadrante más débil (FA) combina las debilidades y las amenazas que enfrenta el destino turístico. Este proceso arrojo algunas interesantes estrategias que se sintetizan más adelante en la tabla 3, entre ellas: puesta en valor de los recursos patrimoniales (F2, F9, O2, O5), diversificación e innovación de productos (F1, F2, F3, O1, O4, O5), programas de capacitación y profesionalización (F5, F6, F7, O1, O2); calidad turística (D6, D7, O6, O7 ), marketing turístico (D8, D9, O4, O5); redes de colaboración (F7, F8, F9, A4, A5) y reestructuración de los planes de desarrollo (A1, A3, D1, D12).

Tanto la realidad turística de la provincia, así como, las respuestas de los principales actores del ramo a una batería de seis preguntas fue la base para identificar las líneas estratégicas y los principales aspectos que deberían abordar los nuevos planes turísticos para las comunidades de Santa Elena. 
Gráfico 1 Opinión de actores turístico sobre el direccionamiento de los planes de turismo

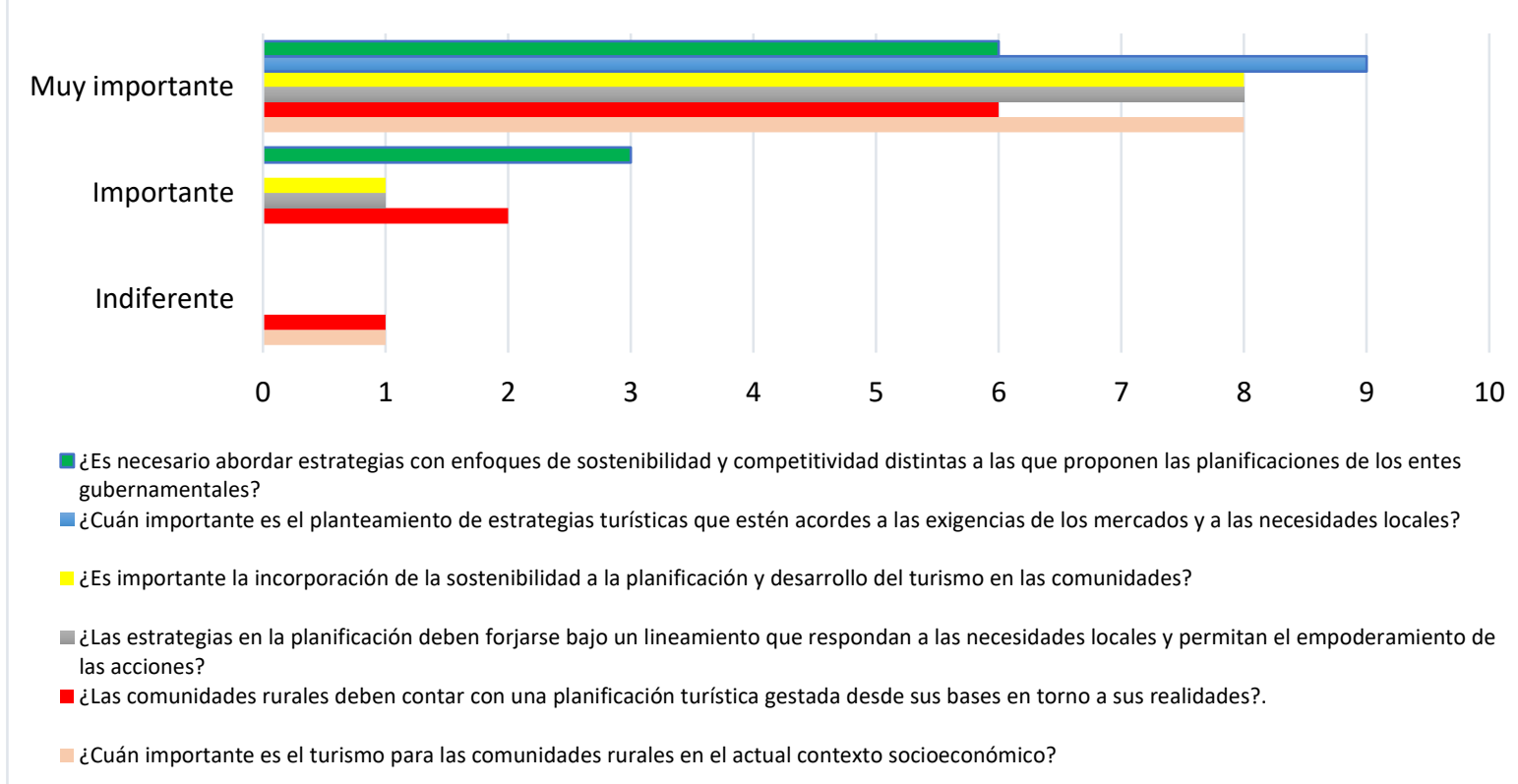

Fuente: Elaboración propia a partir de entrevistas a actores turísticos de la provincia. 2018

A raíz de catalogar como muy importante el abordar aspectos de sostenibilidad y participación local en los nuevos planes de turismo, el grupo focal luego de analizar las concordancias en las preguntas, presenta las consideraciones a manera de líneas estratégicas que deben incorporarse para enfrentar la actividad.

\section{Líneas estratégicas que deben afrontar la planificación turística en comunidades rurales de Santa Elena.}

La revisión de datos secundarios, las entrevistas a los principales actores del turismo, así como, la postura de expertos del grupo focal, concuerdan en las tendencias de un turismo más justo y responsable, que busca destinos no tradicionales y convivir en ellos, con principios de sostenibilidad e incluyentes; lo que deja en evidencia que es preciso afinar los planes de desarrollo turístico, dando enfoques participativos y de sostenibilidad.

Como resultado del trabajo focal, basados en los análisis de los planes de desarrollo existentes, en el FODA del turismo provincial y en los resultados de las entrevistas a los actores, se identificaron y consensuaron variables claves para afrontar la planificación turística en comunidades rurales del litoral santaelenense a través de 7 líneas estratégicas que han de abordarse a partir de 29 aspectos claves y una plataforma transversal que este inmersa y sea considerada en todos los ejes estratégicos.

Tabla 3 Líneas estratégicas y aspectos que deben afrontar los planes turísticos en Santa Elena.

Líneas Estratégicas $\quad$ Aspectos de interés para la planificación turística en comunidades de Santa Elena 


\begin{tabular}{|c|c|c|}
\hline 1 & Recursos naturales & $\begin{array}{l}\text { - Protección y readecuación paisajística } \\
\text { - Gestión y creación de áreas protegidas comunales } \\
\text { - Puesta en valor de los recursos naturales }\end{array}$ \\
\hline 2 & Recursos culturales & $\begin{array}{l}\text { - Rehabilitación y puesta en valor de los espacios paleontológicos y arqueológicos } \\
\text { - Rescate cultural y su puesta en valor }\end{array}$ \\
\hline 3 & $\begin{array}{l}\text { Zonificación } \\
\text { territorio }\end{array}$ & $\begin{array}{l}\text { - Estudios de potencialidad e inventarios turísticos } \\
\text { - Zonificación territorial de espacios } \\
\text { - Usos de los espacios zonificados a través de planes de manejo } \\
\text { - Propuesta de tipologías de turismo en relación a la potencialidad geográfica } \\
\text { - Infraestructuras y equipamientos colectivos }\end{array}$ \\
\hline 4 & $\begin{array}{l}\text { Oferta } \\
\text { alojamiento } \\
\text { restauración }\end{array}$ & $\begin{array}{l}\text { - Diversificación e innovación } \\
\text { - Mayor estancia } \\
\text { - Profesionalización y bilingüismo } \\
\text { - Cualificación y certificación } \\
\text { - Restauración con productos gastronómicos locales }\end{array}$ \\
\hline 5 & Servicios turísticos & $\begin{array}{l}\text { - Artesanías y ferias locales } \\
\text { - Actividades culturales } \\
\text { - Servicios de transporte } \\
\text { - Servicios especializados de turismo }\end{array}$ \\
\hline 6 & $\begin{array}{l}\text { Redes } \\
\text { colaboración }\end{array}$ & $\begin{array}{l}\text { - Asociatividad y cooperación entre comunidades } \\
\text { - Autogestión y protagonismo de las comunidades } \\
\text { - Complementación territorial y uso de los bienes comunes } \\
\text { - Colaboración con entidades locales y con el tejido empresarial }\end{array}$ \\
\hline 7 & Marketing & $\begin{array}{l}\text { - Diversificación de productos turísticos } \\
\text { - La distribución en los mercados } \\
\text { - Atraer y mantener segmentos } \\
\text { - Posicionamiento y generación de imagen en los mercados } \\
\text { - Promoción y difusión de los destinos } \\
\text { - Fidelización de clientes }\end{array}$ \\
\hline & $\begin{array}{l}\text { aforma transversal de } \\
\text { enibilidad }\end{array}$ & $\begin{array}{l}\text { Los procesos que se emprendan en cada estrategia, necesariamente deberá tener } \\
\text { una gestación y empoderamiento de los actores locales, debiendo ser las acciones } \\
\text { continuas y flexibles, que respondan a los intereses endógenos de las } \\
\text { comunidades, cuya oferta deberá ser autentica, donde prime el turismo Inclusivo } \\
\text { que respete la cultura, valores y modos de vida de la comunidad, done la actividad } \\
\text { turística sea responsable y genere la preservación y minimización de impactos, con } \\
\text { constante gestión y educación ambiental, que permita generar una cultura turística } \\
\text { que conlleve a la satisfacción de las experiencias del cliente. }\end{array}$ \\
\hline
\end{tabular}

Fuente: Elaboración propia a partir de entrevistas a actores del turismo y trabajos de grupo focal.

Cada estrategia aborda un número de aspectos clave de interés para el desarrollo del turismo en comunidades, según manifiesta Prado (2012), los aspectos clave dan dirección, que sin ellas la planificación carece de horizontes claros [18]. Las estrategias identificadas se enmarcan en el contexto de la realidad de la provincia, en escenarios turísticos contemporáneos y escenarios futuros que pueden precisar el direccionamiento de la actividad turística.

Las estrategias uno, dos y tres permitirá alternativas turísticas, a su vez, generará protección y uso de los recursos patrimoniales, permitiendo a las comunidades emprender en iniciativas asociadas a la nueva ruralidad. El enfoque de las estrategias cuatro, cinco y seis, están relacionadas a los servicios y productos orientados para el visitante y a la implicación de la población local en la actividad; la estrategia siete responde a las exigencias del mercado turístico con segmentos cada vez más exigentes y, a la competencia dinámica e innovadora. Finalmente la plataforma transversal de 
sostenibilidad se enmarca en los principios de sostenibilidad relacionados con lo social, ecológico, económico y de gobernanza, que necesariamente deben incluirse transversamente en la planificación y en cada uno de los ejes estratégicos.

\section{Discusión.}

El turismo como un sector importante de la economía de un país necesita ser planificado, para realizar proyecciones que determinarán decisiones acertadas y convenientes, he aquí la importancia de la planificación como encaje de previsión que permite incorporar medidas pertinentes en el presente y que tributarán a un futuro adecuado, aunque en la práctica el modelo de planificación establecido en la provincia de Santa Elena no alcanza los estándares esperados.

Las diversas planificaciones y entre ellas la de Santa Elena, tienen como visión utilizar al turismo como motor económico, por lo que en su totalidad plantean acciones hacia la demanda turística y hacia la oferta y sobre ellas las estrategias. Más los aspectos de competitividad y sostenibilidad, aun no se asume como fundamental en el sector turístico, al igual que las implicaciones de otros sectores.

Es preciso enunciar que tanto en las tareas como en la metodología formulada en los planes analizados, existe una carencia de participación de actores comunitarios como dueños de los principales recursos en el sector. En el contexto actual, la participación de estos en los consensos es preponderante ya que produce el empoderamientos de las acciones; además es evidente que en Santa Elena no se considera el aspecto de la zonificación, más aún cuando los enfoques de desarrollo deben apuntar a procesos que se reviertan en el espacio geográfico local.

La planificación requiere considerar no solo a los actores exógenos sino a los elementos endógenos del territorio, así como también gestionar los impactos del desarrollo turístico, de modo que éste promueva el progreso socioeconómico del área y su población.

\section{Conclusiones.}

La planificación en Santa Elena, ha estado orientada hacia el crecimiento económico como consecuencia de la imposición del sistema, políticas y visión gubernamental, careciendo de resultados contundentes en el desarrollo por falta de compromisos locales.

> Los distintos criterios identificados para una planificación del turismo sustentable, no se evidencian en los distintos planes de desarrollo turístico formulados para el territorio de Santa Elena, aunque en el caso de planes parroquiales y el comunitario, se puede extraer aspectos que permita generar bases para los nuevos planes, donde la participación de la comunidad y de los distintos actores permita la gestación y empoderamiento del proceso ligado a la sostenibilidad. 
Mientras menor es la dimensión de la planificación, esta incluye aspectos de sustentabilidad relacionada con lo cultural, ambiental y económico, dando mayor importancia a la participación de los diferentes actores del turismo.

$>$ Una propuesta de planificación turística transversal que apuesta por la integración de los objetivos de desarrollo local, competitividad turística y sostenibilidad territorial es urgente, la consideración de un enfoque multidisciplinar participativo, que considere los patrimonios comunitarios e iniciativas de gestación y empoderamiento, hará de las planificaciones turística distintas a las que en esta oportunidad se ha analizado.

\section{Referencias Bibliográficas.}

[1] DENMAN, Richard. Por un turismo más sostenible. Guía para responsables políticos. Madrid, 2006. ISBN 978-92-844-1189-4 Ed. Programa de las Naciones Unidas para el Medio Ambiente (PNUMA), Organzación Mundial del Turismo (OMT).

[2] PLUMED LASARTE, Marta; GOMEZ BRUNA, Diana y MARTÍN DUQUE, Clara. Planificaciòn turìstica, promociòn y sostenibilidad ambiental: el caso de España. Retos Revista de Ciencias de la Administraciòn y Economìa: ISSN 1390-8618, Volumen VIII (15): 7 - 18, 2018. [Consulta: 10 de Septiembre de 2018]. Disponible en: https:/doi.org/10.17163/ret.n15.2018.01

[3] POPOVICH, María y TOSELlE, Claudia. Planificaciòn de una estrategia participativa y comunidad local. Desarrollo de un destino turìstico en Argentiva. Cuadernos de Turismo, $\mathrm{n}^{\circ}$ 17, 167 - 188, 2006. ISSN: 1139-7861

[4] VERA REBOLLO, José Fernando. Planificaciòn y gestiòn del desarrollo turìstico sostenible: propuestas para la creaciòn de un sistema de indicadores. Documento de trabajo. RUA - Instituto universitario de geografia. Universidad de Alicante. $\mathrm{N}^{\circ}$ 1. 2001. ISSN 1578-679X.

[5] RIVAS GARCIA José. Planificaciòn turìstica y desarrollo sostenible. Septem Ediciones. 2014. 216 p, ISBN: 9788496491649

[6] GARCIA HENCHE, Blanca. Marketing del Turismo Rural. Segunda Edición. Editorial Piramide. 2003. 320 p, ISBN: 9788436820096

[7] INSKEEP, Edward. Planificaciòn turìstica nacional y regional. Madrid: Ed. OMT. 1991. ISBN-10: 047129392x

[8] LÓPEZ PALOMEQUE, Francisco [et al]. Anàlisis territorial del turismo. Barcelona: Editores Ariel, 1997. ISBN: 84-344-3455-5.

[9] HARVEY, David. Rebel Cities, from the right to the city to the urban revolution. London: Editorial: Verso Book 2012. ISBN: 9781844678822 
[10] GOÑI MAZZITELLI, Adriana. Planificaciòn colaborativa: Consolidando Territorios Emergentes en Uruguay. En Seminario Internacional de Investigación en Urbanismo. "VII Seminario Internacional de Investigación en Urbanismo, Barcelona-Montevideo, junio 2015". Barcelona: DUOT, 2015. ISSN2339-6598

[11] BEEBEEJAUN, Yasminah. Participation by the people what will it achieve for the people. England, Town an Country Planning. The journal of the Town and Country Planning Association vol 81 N5, 2012. ISSN 0040-9960

[12] ECUADOR. Gobierno Provincial Autónomo Descentralizado de Santa Elena. Resumen ejecutivo plan de desarrollo y ordenamiento territorial provincial. Santa Elena, 2015.

[13] ECUADOR. Gobierno Autónomo Descentralizado del Cantón Santa Elena. Plan de desarrollo y ordenamiento territorial cantòn Santa Elena. Santa Elena, 2014.

[14] DIEGUEZ CASTRILlÒN, María; GUEIMONDE CANTO, Ana; SINDE CANTORNA Ana y BLANCO CERRADELO, Lidia. Anàlisis de los principales modelos explicativos de la competitividad de los destinos turìsticos en el marco de la sostenibilidad. Revista CULTUR-Revista Cultura e Turismo, 2(5), 101 124, 2015. Disponible en UESC - Universidade Estadual de Santa Cruz(http://www.uesc.br . ISSN-e: 1982-5838

[15] CORNEJO GANGA, Luz Elena. Planificaciòn turìstica sustentable en la regiòn de Coquimbo. Evaluaciòn y aportes metodològicos. Cuaderno de invetigaciòn urbanìstica (65), 15 - 34, 2009. ISSN: 1886-6654

[16] ECUADOR. Ministerio de Turismo de Ecuador - MINTUR). Cordinaciòn General de Estadistica e Investigaciòn Indicadores turìsticos: informaciòn relevante del turismo en Ecuador. Quito, 2017.

[17] PONCE TALANCÓN, Humberto. La matriz FODA: una alternativa para realizar diagnósticos y determinar estrategias de intervención en las organizaciones productivas y sociales. Revista académica Contribuciones a la Economía, 1-16, 2006. ISSN 16968360

[18] PRADO TOBO, Marcela. Prospectiva turìstico sostenible para el municipio de Palmira (valle del Cauca, Colombia) horizonte 2014. Andalucia: Ed. Universidad Internacional de Andalucia. 2012. ISBN 978-84-7993-979-3

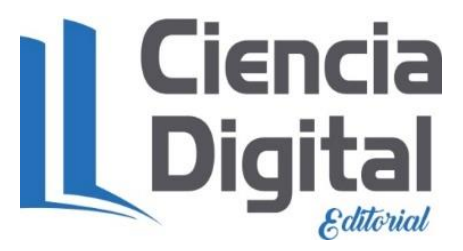




\section{Para citar el artículo indexado.}

Yumisaca J., Delgado A., Pérez M. \& Mendoza A. (2019) Planificación turística sostenible: las comunidades de Santa Elena. Revista electrónica Explorador Digital 3(3), 38-50. Recuperado desde:

http://cienciadigital.org/revistacienciadigital2/index.php/exploradordigital/article/view/442/9 $\underline{96}$

\section{Ciencia \\ Digital \\ Editorial}

El artículo que se publica es de exclusiva responsabilidad de los autores y no necesariamente reflejan el pensamiento de la Revista Explorador Digital.

El articulo queda en propiedad de la revista y, por tanto, su publicación parcial y/o total en otro medio tiene que ser autorizado por el director o editor de la Revista Explorador Digital.
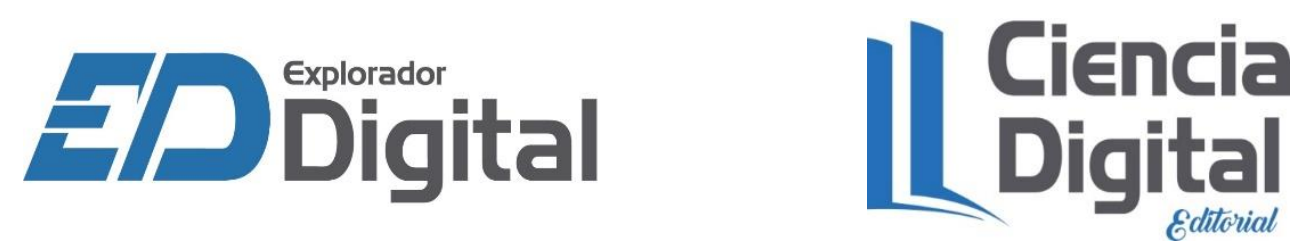